\title{
Look Back and Look Forward: The Growth of Astronomy in Japan
}

\author{
Saeko S. Hayashi ${ }^{1,2,3}$ \\ ${ }^{1}$ TMT-Japan Project Office \\ National Astronomical Observatory of Japan, National Institutes of Natural Sciences \\ email: saeko@naoj.org \\ ${ }^{2}$ Department of Astronomy \\ SOKENDAI (Graduate University for Advance Studies) \\ ${ }^{3}$ Astronomical Society of Japan
}

\begin{abstract}
Astronomy in Japan has long history and has its own "light". The foresight of leaders in the early 20th century helped Japan to become a founding member of the International Astronomical Union. In this paper, a brief description will show the growth in its more recent history with particular emphasis on two important issues: large research facilities and women participation. On the ground or above, the large observational facilities are nowadays outside of the land of Japan, not only in terms of physical presence but also the community they serve. Domestic membership of the Astronomical Society of Japan at the same time is expanding, thanks to the increased opportunities for women. Continuing global cooperation with more diverse composition of members are the keys for sustaining the growth to carry the astronomy in Japan with IAU into the next century, and hopefully to another planet.
\end{abstract}

Keywords. Development, sustainability of astronomy, growth of astronomy, history of astronomy, astronomy in Japan, astronomical collaboration

\section{History as a Backdrop - Looking Back}

Due to its geographical isolation, Japan as a nation has been hiding from the rest of the world. However, this is no longer the case. Let's look back to see what affected the current situation of astronomy in Japan.

Records of observing the sky in Japan go back at least several to ten thousand years ago (Fig. 1). For example, some of the huge rocks in the Kanayama ruins show the conspicuous configuration composed of several stars, and some are thought to be used to idenfity summer and winter solstices. It was important to know the seasons for agriculture, hunting, fishing etc., all are critical for human survival. Even though Japan is isolated from the rest of the world, there has been of course a long-standing influence from China, the nearest powerful country. In earlier years, calendars came from there. Aristocrats, generals, and the imperial house or government hired professionals to improve the calendars with their own observations to use in Japan.

In 1868, power transfered from the feudal system to the imperial system (which itself had a long-standing presence) called Meiji Restoration. At the same time the closed-door policy ended and Japan started rapid industrialization. In 1908, the Astronomical Society of Japan (ASJ) was founded, and in 1919 Japan became a founding member of the IAU, one of the seven (because Japan was not one of the "central" allies at that time). In 1920 the Oriental Astronomical Association of Japan (OAA) was founded. Then during the 1930s to 1940s Japan as a nation took a wrong turn. When World War II ended in 1945, recovery from the war and re-integration into the world's countries started. In 1949 the 


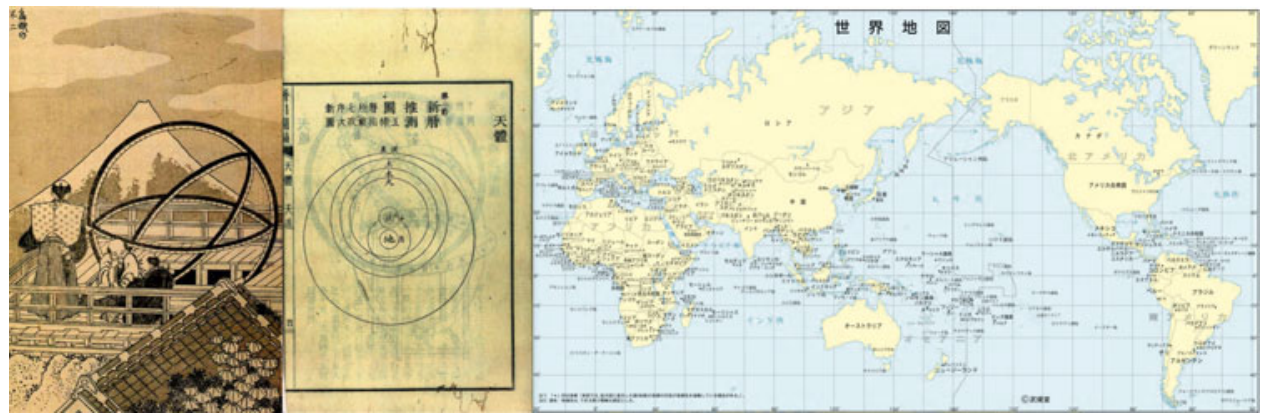

Figure 1. Left: Observation during Edo Era in Japan (Credit: Chiba City Museum of Art) and the concept of the Universe with five planets (Credit: NAOJ), while Galileo was using his telescope. Right: Japan is located close to the centre, hence easier to find in this world map quoted from the Office of Statistics Japan.

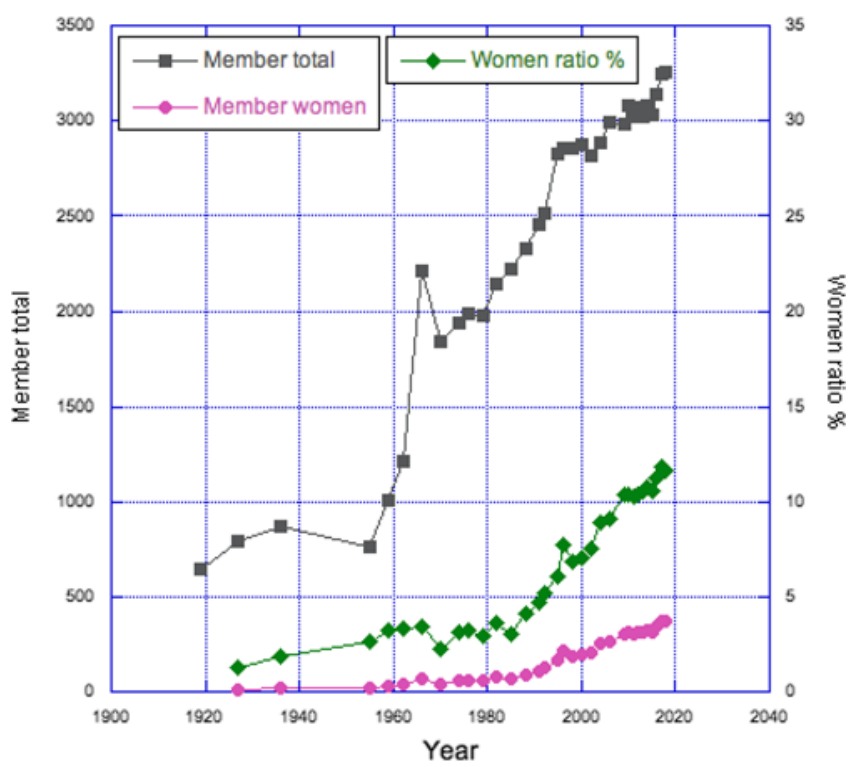

Figure 2. Increase in the membership of the Astronomical Society of Japan. Women's membership started to soar from mid-1980s. (Motizuki \& Kaifu 2016)

reorganized Science Council of Japan started and this SCJ is the adhering member of the IAU. The ASJ is one of the two agencies recognized by the SCJ in the astronomy field. The other association is the Japanese Society for Education and Popularization of Astronomy (JESPA; established in 1989 and incorporated in 2018).

In 1981 Japan signed the United Nations "Convention on the Elimination of all forms of Discrimination Against Women" due to the pressure from outside, and large astronomical facilities started to be built from the 1980s. In 1985 the Equal Employment Opportunity Law took effect at the end of United Nations Decade for Women (1976 to 1985). Around that time, Japanese labour law started to allow night work by women. From around the 1990s, women's participation became more visible, preceded by the case in the ASJ where the women's ratio started to show significant growth in the mid-1980s (see Fig. 2). 


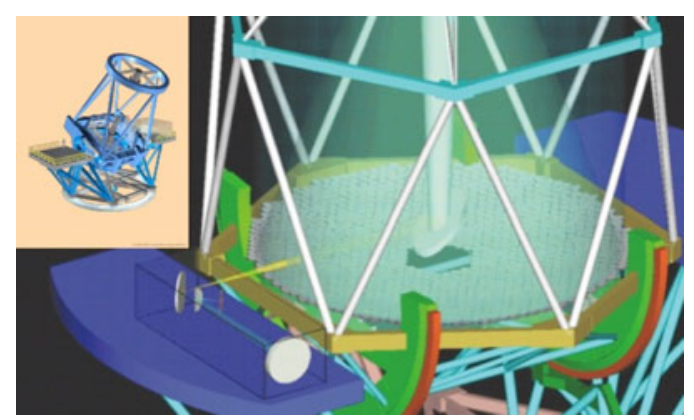

Figure 3. 8.3-m Subaru Telescope to the upper left - one of the current largests telescopes, with the 30-m Thirty Meter Telescope (TMT). (Both numbers refer to the physical size. $8.2 \mathrm{~m}$ is the aperture of the Subaru Telescope.) The larger one is for you, the junior members newly inducted to the IAU.

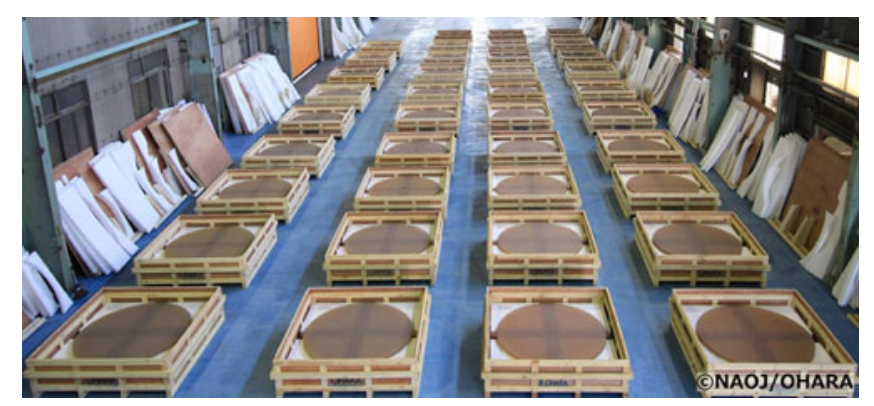

Figure 4. Crearceram glass ceramics blanks are in full production for TMT's primary mirror in Japan. These 1.52-m circular discs go through stages of grinding, aspherical polishing, hexing, mounting before going overseas for final touch up, and eventually to the telescope site. TMT needs 594 of such segments. This is another example of utilizing what is available in the industry to specialize to match the research needs.

\section{Actions being taken: Case of the Astronomical Society of Japan}

After World War II, researchers were able to return to their workplace and carry out their research. The financial support was and still is a real struggle, yet certain freedom is intact. Large research facilities started to appear, as a conduit for stimulating Japanese industry. Astronomy facilities are no exception. Developing highly sensitive sensors, precise motion of a telescope mount, active feedback of the support structure, are among many of the demands that were made during the construction of such facilities. Here is a brief and incomplete list of such facilities that foreign researchers may recognize. Then those facilities helped the increase in the number of astronomers and astronomyrelated professionals, as shown in Fig. 3 .

Large Facilities

- ALMA (Atacama Large Millimeter Array) is a joint effort of three regional partners Europe's European Southern Observatory (ESO), Japan's NAOJ and North America's National Radio Astronomical Observatory (NRAO);

- Subaru Telescope (Fig. 4) was funded by Japanese taxpayers through NAOJ. Already international collaborations took place in its instrumentation programmes. It is going to extend its operation with a multi-countries' partnership; 


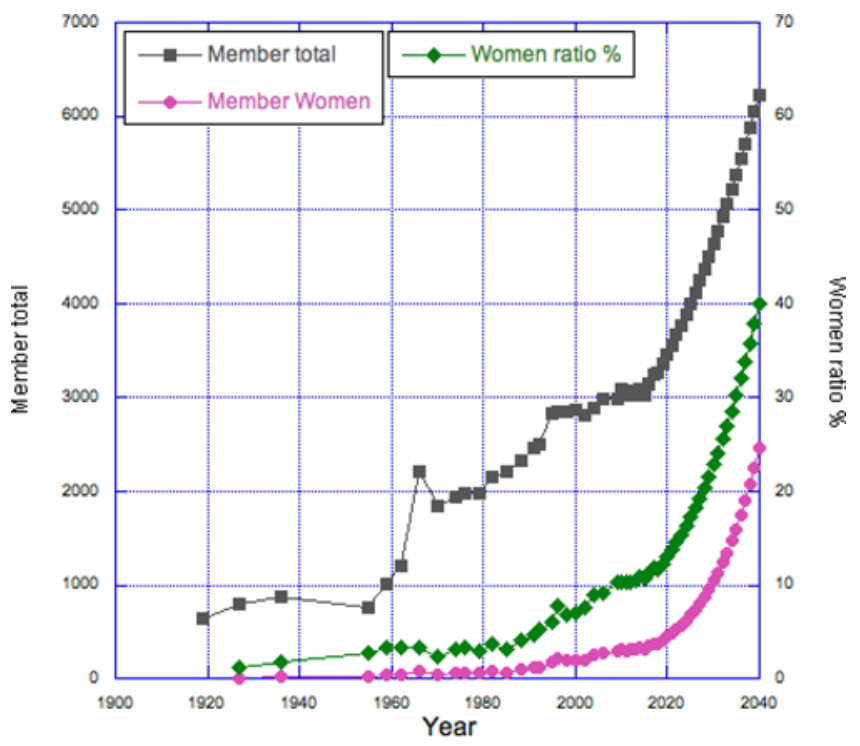

Figure 5. If the membership in ASJ - a good indicator of the numbers of astronomers in Japan - increases at a rate of recent pace, let's say the optimistic $3 \%$ in overall numbers and another optimistic rate of $9 \%$ in women after Motizuki \& Kaifu (2016), the women's participation will reach $30 \%$ by the mid-2030s. This diagram is more of author's dream than a true scholastic prediction.

- KAGRA (Kamioka Gravitational Wave Detector) is undergoing the assembly, integration, and verification phase. Soon it will become the third station of the global network of gravitational wave detectors, after LIGO and VIRGO;

- Kamiokande (Kamioka Nucleon Decay Experiment) and Super Kamiokande are the neutrino detectors deep inside the mountain. Their detectors were provided by Hamamatsu Photonics, another good example of a partnership between academia and industry;

- VSOP (VLBI Space Observatory Programme) mission is led by the Institute of Space and Astronautical Science (ISAS/JAXA), in collaboration with NAOJ. The HALCA (Highly Advanced Laboratory for Communications and Astronomy) satellite was launched Feb. 12, 1997 and successfully deployed its 8-metre antenna on February 27-28, 1997. This space mission portion ended Nov. 30, 2005.

There have been smaller scale collaborations which led to larger ones. Here follows a short example list.

Local Collaborations - for details, see Kaifu (2019) in this volume.

- UKIRT, JCMT (Japan-UK collaboration in 1980-1990s)

- EAVN (East Asia VLBI Network)

- 1990- EACOA (East Asian Core Observatories Association): meeting (EAMA), fellowship, young astronomers meeting (EAYAM)

The upward trend of the membership of the ASJ (Fig. 5) became more distinct from the mid-1980s when the women's participation showed a significant increase. And the women's enrolment rate is getting higher. One of the reasons is the involvement of graduate students. In 1997, a new membership category for student was created in the ASJ membership, and la arge number of eligible members moved from being associate 
members to full members. The younger generation has a better proportion of women, which is helping the increase of the women's enrolment in the ASJ.

The childcare room was created from 1997 during the biannual meeting of the ASJ. Any member of the ASJ participating in the meeting can bring infants and young children to the meeting without worrying about making childcare arrangement themselves. They pay a nominal (hence affordable) fee. This system was established much earlier than in other academic associations, thanks to the foresight of the leaders. This was not only for women. Due to the scarcity of jobs, many young researchers had to rely on a spouse's income and shared the child-caring responsibility, unlike the rest of Japanese society. In addition, special sessions for and by young researchers and women during the biannual meeting helped create and enhance the networking, thereby empowering them with appropriate information.

Recently there are more women taking leadership positions and getting awards in the ASJ. One of the two Vice-President positions has been a woman from 2012, and women are serving as committee members and even chairs. Of particular importance would be one member in the selection committee since 2017. Recipients of the ASJ awards include prominent women astronomers in Japan. Examples are Chushiro Hayashi Award (started 1996) to Mariko Kato in 2003, PASJ Award (started 1996) to Kyoko Matsushita in 2002, and to Misato Fukagawa in 2017.

One of the cornerstone projects of ASJ 100 was the textbook series for college-level students in Japanese. The content is greatly improved from the previous series, with many more demonstrations of Japanese researchers' contributions in the advancement of the field of astronomy.

\section{Outcome (or a hint of it) - Looking Forward}

One good example for the near-future direction would be the Thirty-Meter Telescope (TMT) project which involves five countries - USA, Canada, Japan, China and India. In Japan, NAOJ under the National Institutes of Natural Sciences (NINS) has responsibility for producing the primary mirror blanks, polishing a significant number of them, the telescope structure and instruments, in a close working relation with companies. Since it takes a long time for this scale of a telescope to be built, many of the staff working on this project won't be able to stay in their position to see its first light. Nevertheless, it is very important to carry out this project and ensure the highest possible performance, so that the next generation can open the brand-new fields of astronomy, even ones that we are not thinking of now.

Already taking place is the production of the blanks for the primary mirror. Like other big telescopes, making the primary mirror takes the longest time. According to the plan, casting all the blanks will take five more years. The polishing process will be in four countries - Japan, USA, India and China. The mileage travelled by these mirror segments will surely be a large number in the end.

Naturally the staff working on-site at this telescope will be more diverse - being on the land far from their home institutions. And this new generation will be well trained to be conscientious about their surroundings, namely the environment, the local community and its traditions. I would like to state that by the time the telescope is built, the women's participation in astronomy in Japan will be close to $30 \%$.

\section{Discussion}

EkERs: The business model in Japan in terms of big telescopes is different from other countries. Collaboration between the academia and the companies looks good when seeing from outside. What is the reason? 
HAYASHI: That is what I hear. However I do not have a good explanation. One obvious reason is that, by collaborating with highly demanding reseachers building for example a big telescope, comapnies gain valuable techniques that later become applicable to their commodity products.

\section{References}

Kaifu, N. 2019, IAU Symposium 349, these Proceedings

Motizuki, Y. \& Kaifu, N. 2016, The 10th East Asian Meeting on Astronomy, held in Seoul, Korea in 2016, astro1.snu.ac.kr/eama10 